\title{
Carcinoma de Pulmão de Células não Pequenas
}

\author{
Non-small cell lung cancer
}

\section{INTRO DUÇÃO}

Em 1999, o percentual demortalidade por câncer de pulmão foi de $12,37 \%$, o maior dos 114.235 mil óbitos por câncer registrados naquele ano. De acordo com as estimativas da incidência e mortalidade por câncer no Brasil para 2002, do Instituto $\mathrm{N}$ acional de C âncer - IN C A/M inistério da Saúde, esse tumor somaria 21.425 casos novos e 14.127 óbitos, vindo a ser a primeira causa de óbito por câncer entre os homens e a segunda, entre as mulheres.

0 tabagismo é o maior responsável por essa doença e a sua ação é tão maléfica, que, mesmo se todos os fumantes parassem com seu vício imediatamente, o número de casos de câncer do pulmão não diminuiria nos próximos 20 anos.

Apesar de todo o avanço verificado no tratamento das neoplasias, a sobrevida em cinco anos dos pacientes com câncer de pulmão de células não pequenas permanece de aproximadamente $14 \%$.

Esses dois aspectos de um mesmo problema enfatizam a importância que tem a prevenção primária, pelo combate sistemático ao tabagismo, como a principal ação de saúde pública, capaz de efetivamente reduzir tanto a incidência como a mortalidade por câncer de pulmão entre uma dada população.

A baixa eficácia do tratamento, por sua vez, tem como um dos problemas identificados o fato de a grande maioria dos casos apresentar-se com doença avançada ao diagnóstico e de somente de $25 \%$ a $30 \%$ de todos os casos de câncer inicial de pulmão serem curáveis pelo tratamento cirúrgico.

Embora a radioterapia pós-operatória diminua a ocorrência da recidiva local, nenhum estudo randomizado demonstrou impacto positivo na sobrevida dos pacientes operados. 0 uso de radioterapia adjuvante, nos casos de ressecção completa de doença tumoral classificada como T 3 ou N 2, tem demonstrado melhoria no controle local da neoplasia, mas também sem apresentar impacto na sobrevida dos doentes.

N os casos em estádio patológico I, II e III, não existe evidência de que a quimioterapia adjuvante tenha impacto na sobrevida de pacientes que se submeteram a ressecção cirúrgica completa.

Em relação à quimioterapia neoadjuvante, os resultados são mais encorajadores, demonstrando uma melhoria estatisticamente significante na sobrevida dos pacientes que receberam quimioterapia pré-operatória.

0 estádio IIIB inclui pacientes que não são candidatos a tratamento cirúrgico, pelo menos inicialmente, por causa de doença localmente avançada (T 4 ou N 3). É provável que a quimioterapia concomitante à radioterapia melhore a sobrevida desses pacientes (como em outro estágio tumoral), quando comparado com o uso de radioterapia curativa (exclusiva); contudo não existe comprovação desse fato.

A quimioterapia paliativa melhora a sobrevida de doentes com carcinoma avançado de pulmão, mas esse benefício parecer ser restrito a doente com capacidade funcional (PS - Perfomance Status) igual ou maior do que $70 \%$ (Karnofsky), que corresponde ao índice 0 ou 1 (Zubrod).

\section{EXAMES DE AVALIAÇÃO}

\author{
CONFIRMAÇÃO DIAGNÓSTICA \\ - Anamnese; \\ - Exame físico; \\ - Citologia de escarro; \\ - Citologia de líquido pleural; \\ - Broncoscopia com biópsia ou lavado; \\ Punção por agulha fina ou biópsia de \\ linfonodo(s); ou $P$ \\ - Punção biópsia pulmonar e Exame cito- ou
}


histopatológico, conforme a natureza do material coletado.

\section{CONDIÇÕES CLÍNICAS DO DOENTE}

Exames de laboratório

- Sangue - H emograma completo;

- Sangue - Dosagem de glicose, uréia, creatinina, sódio, potássio, cálcio, fosfatase alcalina, gama GT e DH L;

- Sangue - Tempo e atividade de protombina e tempo parcial de tromboplastina;

- Urina - EAS;

- Fezes - Parasitológico.

\section{Avaliação da função respiratória}

A avaliação funcional respiratória é realizada em pacientes el egíveis para tratamento cirúrgico e inclui a espirometria e gasometria arterial. A cintilografia pulmonar perfusional e o teste ergométrico com medida do consumo de oxigênio (V 02) são reservados para pacientes de alto risco cirúrgico.

\section{EXTENSÃO DA DOENÇA}

Exames Radiológicos

- Radiografia simples de tórax em PA e perfil;

- Tomografia computadorizada de tórax e do abdome superior;

- Ressonância magnética - Indicada em casos selecionados, para o diagnóstico diferencial entre tumor (ou recidiva tumoral) e cicatriz residual; para o diagnóstico diferencial das atelectasias pós-obstrutivas; na avaliação dos tumores do ápice pulmonar; para a determinação do envolvimento do plexo braquial; na invasão da região paravertebral; e na invasão mediastinal para avaliar a relaçãa entre o tumor e a parede torácica, o coração e os grandes vasos.

Cintilografia Ó seas solicitada nos casos de doentes com dor óssea ou el evação da fosfatase alcalina. Q uando o resultado for anormal, mas sem padrão compatível com metástases ósseas, faz-se necessário 0 esclarecimento diagnóstico, através de exame de radiografia simples ou de tomografia computadorizada de região esquelética cintilograficamente anormal.
Tomografia computadorizada de crânio: indicada para doentes com suspeita clínica de acometimento do sistema nervoso central (cefaléia, tonteiras, mioclonias, convulsões...).

Punção por agulha fina ou biópsia de linfonodos: quando houver linfonodo(s) palpável(eis), quejá não tenha(m) sido a fonte do diagnóstico patológico.

Mediastinoscopia: indicada na presença de imagem inicial, à tomografia computadorizada, de lesões centrais ou linfonodos mediastinais maiores que $10 \mathrm{~mm}$ no seu menor diâmetro. Também está indicada no caso de doença metastática e tumor primário ressecáveis, devendo ser realizada antes do tratamento do tumor primário e das metástases do cérebro ou da supra-renal.

Mediastinotomia para-esternal esquerda: indicada para a avaliação de tumores do lobo superior ou quando houver suspeita radiológica ou tomográfica de linfonodo maior que $10 \mathrm{~mm}$ no seu menor diâmetro, no nível 5 ou 6.

Toracoscopia: indicada para doentes com tumor de pulmão ressecável e derrame pleural com citologia negativa para malignidade.

Biópsia da lesão metastática: quando existir suspeita de acometimento do fígado, osso ou supra-renal.

\section{ESTADIAMENTO}

Feito conforme a 5a Edição de TNM Classificação de Tumores M alignos - UICC, 1997; M inistério da Saúde, 1998 (disponível em www.inca.gov.br, página do INCA na Internet).

\section{TRATAMENTO}

\section{DEFINIÇÃO DAS MODALIDADES TERAPÊUTICAS}

Cirurgia

0 procedimento cirúrgico recomendado

é a Iobectomia ou pneumectomia 
acompanhada de linfadenectomia hilar e me diastinal. O utra ressecção possível é a lobectomia com broncoplastia, que poderá ser empregada em situações específicas e em pacientes com estadiamento $\mathrm{N} 0$.

Ressecções menores que a lobectomia (segmentectomias ou cunha) poderão ser utilizadas em pacientes com reserva pulmonar limítrofe.

A ressecção em bloco compreenderá a ressecção pulmonar juntamente com a linfadenectomia associada à ressecção das estruturas adjacentes (parede torácica, pericárdio e diafragma).

\section{Radioterapia}

Em todas as situações de radioterapia descritas, a retirada da medula espinhal do campo de irradiação é feita quando se atinge a dose de $45 G y$.

\section{Radioterapia Exclusiva (Radical)}

Presença de linfonodos acometidos (N 2

C línico): Radioterapia sobre a lesão primária e sobre as regiões de drenagem linfática mediastinais (ipsilateral e contralateral), com $02 \mathrm{~cm}$ de margem peritumoral.

Ausência de linfonodos acometidos (N O): Radioterapia sobre a lesão primária, com 02 $\mathrm{cm}$ de margem peritumoral e de $01 \mathrm{~cm}$ sobre 0 mediastino.

Acometimento da fossa supraclavicular ipsilateral (N 3 ) ou do ápice pulmonar: Radioterapia sobre a lesão primária (com 02 $\mathrm{cm}$ de margem peritumoral), mediastino contralateral (com $01 \mathrm{~cm}$ de margem) e fossa supraclavicular ipsilateral, todos englobados conjuntamente.

\section{Doses}

- Em todos os casos de radioterapia exclusiva, usa-se a dose inicial de 45 a 50G y em cinco semanas ( 25 frações), na dose diária de 1,8 a $2,0 \mathrm{G}$ y. U tilizados campos paralelos e opostos, ântero-posteriores, com energia de fótons $\geq$ $06 \mathrm{M} \mathrm{v} \mathrm{(acelerador} \mathrm{linear),} \mathrm{podendo} \mathrm{também}$ ser utilizada energia de 1,25 M v (unidade de cobalto), porém dando-se preferência a energias $\geq 10 \mathrm{M} \mathrm{v}$ (acelerador linear), em caso de distância ântero-posterior $\geq 18 \mathrm{~cm}$. Total de campos da dose inicial $=50$ campos .

- A dose de reforço sobre a lesão primária é de 10 a 16G y em 1 a 1,5 semana (05 a 08 frações), na dose diária de 1,8 a 2,0G y, em campos paralelos opostos, ântero-posteriores, com a mesma energia da fase inicial de tratamento, ou com outra combinação decampos, caso o planejamento tenha sido feito em 3D. Total de campos da dose de reforço $=10$ a 16 campos (10 a 24 campos, se planejado em 3D ). Total de campos da radioterapia exclusiva $=60$ a 66 campos (ou 60 a 74 campos, se planejado em 3D ).

Nota - No caso de tumor periférico sem indicação cirúrgica, tratar apenas o tumor com $02 \mathrm{~cm}$ de margem peritumoral, sem englobar o mediastino, até a dose de 60 a 66 Gy em 06 a 6,5 semanas (30 a 33 frações), na dose diária de 1,8 a 2,0G y, em campos paralelos opostos, ântero-posteriores, com a mesma energia acima descrita para dose inicial. Total de campos da radioterapia exclusiva = 60 a 66 campos (ou 60 a 74 campos, se planejado em 3D).

\section{Radioterapia Paliativa}

D estinada aos pacientes com PS entre $30 \%$ e $49 \%$ (Karnofswy) ou PS 3 (Zubrod), isto é, doentes que permanecem no leito mais da metade do dia e necessitam de cuidados de enfermagem. Geralmente, a redução da capacidade funcional se associa também a perda ponderal.

Doença loco-regional presente e sintomática: utiliza-se sempre radioterapia sobre a lesão primária, com $02 \mathrm{~cm}$ de margem peritumoral, em campos paralelos opostos, ântero-posteriores ou látero-laterais (deacordo com a área a ser irradiada), com a energia de fótons $\geq 1,25 \mathrm{M} \mathrm{v}$ (unidade de cobalto ou acelerador linear). D ose: 30G y, em 02 semanas (10 frações), na dose diária de 03 Gy. Total de campos $=20$ campos.

Lesão metastática: radioterapia em campos paralelos opostos, ântero-posteriores ou látero-laterais (de acordo com a área a ser irradiada), com energia de fótons $\geq 1,25 \mathrm{M} \mathrm{v}$ (unidade de cobalto ou acelerador linear). N 0 caso de metástase cerebral irrradiar todo 0 crânio com proteção ocular. D ose: dose única (exceto em cérebro) de 08G y; 20G y em uma semana (05 frações) na dose diária de 04G y; 
ou $30 \mathrm{G}$ y em duas semanas (10 frações) na dose diária de 03Gy. Total de campos $=02$ campos (dose única, exceto em cérebro); 10 campos (em uma semana) ou 20 campos (em duas semanas).

Síndrome de Compressão da veia C ava Superior: radioterapia em campos paralelos opostos, ântero-posteriores, utilizando energia defótons $\geq 6 \mathrm{M} \mathrm{v}$ (acelerador linear), podendo também ser utilizada energia de 1,25 M v (unidade de cobalto). Em caso de distância ântero-posterior $\geq 18 \mathrm{~cm}$, prefere-se a energia de fótons $\geq 10 \mathrm{M} v$ (acelerador linear). D ose: 20G y em uma semana (05 frações) na dose diária de 04G y ou dose única de 08G y. Total de campos $=10$ campos (em uma semana) ou 02 campos (dose única).

\section{Braquiterapia Endobrônquica}

Indicações: embora não controle localmente o tumor não inicial nem impacte sobre a sobrevida dos doentes, a braquiterapia endobrônquica pode ser eventualmente, em casos selecionados, indicada no pós-operatório, quando a margem cirúrgica do coto brônquico estiver positiva; e como al ternativa a radioterapia externa fracionada, com finalidade paliativa, nos casos de obstrução brônquica por tumor ou nos casos de sangramento endobrônquico. $\mathrm{N}$ os casos de obstrução brônquica total, a laserterapia precede a braquiterapia endobrônquica, quando esta é indicada.

Alta taxa de dose (BATD): quando indicada, é feita à dose de 22,5 Gy em 03 frações, e o tratamento é realizado com intervalo de 15 dias entre as frações e o cálculo da dose a $01 \mathrm{~cm}$ da fonte.

Radioterapia e Quimioterapia Concomitantes

A radio-quimioterapia é utilizada nos casos de doentes em estágio III considerados inicialmente inoperáveis ou não candidatos à radioterapia exclusiva, e que se encontrem com a capacidade funcional classificável como PS $\geq 50 \%-69 \%$ (K arnofsky) ou PS $\leq 2$ (Zubrod).

$0 \mathrm{~s}$ aspectos técnicos do planejamento da radioterapia não são alterados pelo uso concomitante da quimioterapia.

Esquema de $\mathbf{Q}$ uimioterapia: $C$ isplatina $50 \mathrm{mg} / \mathrm{m}^{2} /$ dia - EV - nos dias D 1, D 8, D 29 e D 36, associada a Etoposido - $50 \mathrm{mg} / \mathrm{m}^{2} / \mathrm{dia}$ -
EV - nos dias D 1 a D 5 e D 29 a D 33.

Esquema de Radioterapia: Inicia-se no mesmo D 1 da quimioterapia:

a) Tumores do ápice pulmonar - Radioterapia sobre a lesão primária (com $02 \mathrm{~cm}$ de margem peritumoral) e no mediastino contralateral (com $01 \mathrm{~cm}$ de margem) e fossa supraclavicular ipsilateral. D ose: 45G y em 05 semanas (25 frações) na dose diária de $1,8 \mathrm{G}$ y, em campos paralelos opostos, ântero-posteriores, com energia de fótons $\geq 1,25 \mathrm{M} v$ (unidade de cobalto ou acelerador linear). Em caso de distância ântero-posterior $\geq 18 \mathrm{~cm}$, preferese a energia de fótons $\geq 10 \mathrm{M} \mathrm{v}$. Total de campos $=50$ campos.

b) Tumores de outras localizaçõesquenão o ápice pulmonar - Radioterapia sobre a lesão primária (com $02 \mathrm{~cm}$ de margem peritumoral) e no mediastino contralateral $(\mathrm{com} 01 \mathrm{~cm}$ de margem). D ose inicial - 45 a $50 \mathrm{G}$ y em cinco semanas (25 frações) na dose diária de 1,8 a 2,0G y, em campos paralelos opostos, ânteroposteriores, com energia de fótons $\geq 06 \mathrm{M} \mathrm{v}$ (acelerador linear), podendo também ser utilizada energia de 1,25 M v (unidade de cobalto), porém dando-se preferência a energias $\geq 10 \mathrm{M} \mathrm{v}$ (acelerador linear), em caso de distância ântero-posterior $\geq 18 \mathrm{~cm}$. Total de campos da dose inicial $=50$ campos. U tiliza-se dose de reforço sobre lesão primária de 10 a 16G y em 01 a 1,5 semana (05 a 08 frações) na dose diária de 1,8 a 2,0G y, em campos paralelos opostos, ântero-posteriores, com a mesma energia da fase inicial de tratamento, ou com outra combinação de campos, caso o planejamento tenha sido feito em 3D. Total de campos da dose de reforço $=$ 10 a 16 campos (10 a 24 campos, se planejado em 3D). Total de campos da radioterapia exclusiva $=60$ a 66 campos (ou 60 a 74 campos, se planejado em 3D).

\section{Quimioterapia e Radioterapia Seqüencial}

Esta associação terapêutica é utilizada nos casos de doentes em estágio III inicialmente considerados inoperáveis ou não candidatos à radioterapia exclusiva, que se encontrem com a capacidade funcional classificável como PS $\geq 50 \%-69 \%$ (Karnofsky) ou PS $\leq 2$ (Zubrod) ou sejam idosos, masque, por conta de outros problemas de saúde, não tolerem a quimio-radioterapia, que é um tratamento 
mais tóxico.

Esquema de Q uimioterapia: C isplatina $100 \mathrm{mg} / \mathrm{m}^{2} / \mathrm{dia}$ - EV - nos dias D 1 e D 29, e Vimblastina - 05mg/ $\mathrm{m}^{2} / \mathrm{dia}$ - EV - nos dias D 1, D8, D 15, D22 e D 29.

Esquema de Radioterapia: Iniciada no D 50, a radioterapia é aplicada sobre a lesão primária (com $02 \mathrm{~cm}$ de margem peritumoral) e no mediastino contralateral $(\mathrm{com} 01 \mathrm{~cm}$ de margem). D ose: 45 a $50 \mathrm{G}$ y em cinco semanas (25 frações) na dose diária de 1,8 a 2,0G y, em campos paralelos opostos, ânteroposteriores, com energia de fótons $\geq 6 \mathrm{M} \mathrm{v}$ (acelerador linear), podendo também ser utilizada energia de 1,25 M v (unidade de cobalto). Em caso de distância ântero-posterior $\geq 18 \mathrm{~cm}$, prefere-se a energia de fótons $\geq$ $10 \mathrm{M} v$ (acelerador linear). Total de campos = 50 campos. U tiliza-se dose de reforço sobre lesão primária de 10 a $16 \mathrm{G}$ y em 01 a 1,5 semana (05 a 08 frações) na dose diária de 1,8 a 2,0G y, em campos paralelos opostos, ântero-posteriores, com a mesma energia da fase inicial de tratamento, ou com outra combinação de campos, caso o planejamento tenha sido feito em 3D. Total de campos da dose de reforço $=10$ a 16 campos (10 a 24 campos, se planejado em 3D). Total de campos da radioterapia seqüencial $=60$ a 66 campos (ou 60 a 74 campos, se planejado em 3D).

\section{Radioterapia Pós-Operatória}

Radioterapia sobre o tumor residual (com margem de $02 \mathrm{~cm}$ ) e no mediastino ipsilateral (com margem de $01 \mathrm{~cm}$ ). D ose: 60G y em 06 semanas (30 frações) na dose diária de 1,8 a 2,0 Gy, em campos paralelos opostos, ânteroposteriores, com energia de fótons $\geq 6 \mathrm{M} \mathrm{v}$ (acelerador linear), podendo também ser utilizada energia de 1,25 M v (unidade de cobalto). Em caso de distância ântero-posterior $\geq 18 \mathrm{~cm}$, prefere-se a energia de fótons $\geq 10 \mathrm{M} v$ (acelerador linear). Total de campos $=60$ campos.

N ota 1 - N a ausência de tumor residual a dose total de radioterapia pós-operatória, adjuvante, é de $50 \mathrm{G}$ y em cinco semanas ( 25 frações) na dose diária de 2,0G y, em campos paralelos opostos, ântero-posteriores, com energia de fótons $\geq 6 \mathrm{M} \mathrm{v}$ (acelerador linear), podendo também ser utilizada energia de 1,25 $M v$ (unidade de cobalto). Em caso de distância ântero-posterior $\geq 18 \mathrm{~cm}$, preferese a energia de fótons $\geq 10 \mathrm{M} v$ (acelerador linear). Total de campos $=50$ campos.

Nota 2 - No caso de tumor periférico sem acometimento do mediastino, este não é irradiado.

\section{Quimioterapia Neoadjuvante à Cirurgia}

Indicada para pacientes com acometimento mediastinal $\mathrm{N} 2$, comprovado patologicamente, e que se encontrem com PS $\geq 50$ (Karnofswy) ou PS $\leq 2$ (Zubrod).

Esquema quimioterápico - M itomicina $06 \mathrm{mg} / \mathrm{m}^{2}$ - EV - no dia D 1; Ifosfamida $3.000 \mathrm{mg} / \mathrm{m}^{2}$ - no dia D 1; M esna - $20 \%$ da dose de ifosfamida - EV - concomitante à administração da ifoffamida e repetido $04 \mathrm{e}$ 08 horas após; e Cisplatina - 50mg/ $\mathrm{m}^{2}$ - EV no dia D 1. Total de 03 ciclos com intervalo de 21 dias entre eles.

Nota 1 - Encontra-se sob avaliação a proposta de padronização de novo esquema para esta finalidade de quimioterapia.

Nota 2 - No caso de metástase supra-renal com indicação cirúrgica, o esquema de quimioterapia neoadjuvanteé: Cisplatina- 75$100 \mathrm{mg} / \mathrm{m}^{2}$ - EV - no dia D 1, e Etoposido $100 \mathrm{mg} / \mathrm{m}^{2} / \mathrm{dia}$ - EV - do dia D 1 a D 3. Total de 03 ciclos com intervalo de 21 dias entre eles.

\section{Quimioterapia Paliativa}

Reservada para doentes com câncer pulmonar avançado e que se apresentem com PS $\geq 70 \%$ (Karnofsky) ou PS $\leq 1$ (Zubrod).

Esquema quimioterápico: C arboplatina AUC (area under the curve) $=5-E V-$ no dia D 1, e Etoposido - $100 \mathrm{mg} / \mathrm{m}^{2} / \mathrm{dia}$ - EV - do dia D 1 a D 3. a cada 21 dias, num total de 04 ciclos.

\section{TRATAMENTO POR ESTÁDIOS}

\author{
Estádio | E || \\ Ressecção cirúrgica do lobo que contenha \\ o tumor. O casionalmente, é indicada \\ bilobectomia ou pneumectomia. Embora a
}


segmentectomia ou ressecção em cunha possam ser justificáveis, em caso de função pulmonar limítrofe, elas não são preconizadas porque se associam a uma al ta taxa de recidiva local.

A radioterapia pós-operatória (ver os itens Radioterapia pós-operatória e Braquiterapia Endobrônquica) é usada em pacientes com margens cirúrgicas positivas.

A radioterapia radical ou paliativa está indicada nos pacientes não candidatos à cirurgia, conforme as condições clínicas do paciente. As contra-indicações cirúrgicas prendem-se a co-morbidades que 0 doente apresente, especialmente as de natureza respiratória (reserva funcional insuficiente para ressecção pulmonar) ou cárdiocirculatória (isquemia coronariana sem controle) ou ainda em caso de recusa do doente a submeter-se ao tratamento cirúrgico.

Estádio IA (T1NOMO), IB (T2NOMO), IIA (T1N1MO) E IIB (T2N1 E T3NOMO)

Cirurgia exclusiva, consistindo de lobectomia com ressecção dos linfonodos hilares e mediastinais.

Situação especial 1 - Tumor do ápice pulmonar - Radioterapia e quimioterapia concomitantes, seguindo-se de cirurgia.

Situação especial 2 - Tumor com invasão da parede, diafragma ou pericárdio (sem derrame pericárdico) - Ressecção pulmonar, toracectomia e ressecção dos linfonodos hilares e mediastinais.

Situação especial 3 - Tumor com paralisia diafragmática - Se 0 acometimento do nervo frênico for baixo e não houver derrame pericárdico, indica-se a ressecção em bloco da lesão e dos linfonodos hilares e mediastinais; se o acometimento do nervo frênico for alto e por invasão direta do tumor, não se indica o tratamento cirúrgico.

Situação especial 4 - Tumor com paralisia de corda vocal - Se à esquerda e com lesão baixa, faz-se a ressecção em bloco da lesão e dos linfonodos hilares e mediastinais; se à direita e com lesão alta, não se indica a cirurgia, e o caso é classificado como em estádio IIIB.

Estádio IIIA (T1 ou T2N2 e T3N1 ou N2)

T 3N 1M 0 - Cirurgia exclusiva.

T 3N 1M 0 com síndrome de Pancoast Radioterapia e quimioterapia concomitantes, seguido de cirurgia.

T 1 ou T 2 ou T 3N 2 M 0 - Quimioterapia neoadjuvante seguida de cirurgia.

A radioterapia adjuvante é indicada quando confirmado, após a cirurgia, tratarse de $\mathrm{N} 2$ na peça operatória. As radioterapia e quimioterapia concomitantes ou radioterapia radical ou radioterapia paliativa estão indicadas nos pacientes não candidatos à cirurgia conforme respectivamente especificado no sub-item "D efinição das M odalidades Terapêuticas".

Estádio IIIB (T4 ou N3)

T4 sem derrame pleural ou N 3 (PS 0 e 1) Radioterapia e quimioterapia concomitantes. T4 sem derrame pleural ou N 3 (PS 2) Q uimioterapia e radioterapia seqüencial.

T4 com derrame pleural - Quimioterapia paliativa.

T 4 N 0 com invasão da carina - Considera-se o tratamento cirúrgico em casos selecionados, sendo indicado carinectomia ou braçadeira traqueal com pneumectomia.

Situação especial 1 - Em paciente sem condições clínicas para quimioterapia, indicase a radioterapia radical ou paliativa, na dependência das condições clínicas do paciente.

Situação especial 2 - Síndrome de veia cava superior - Radioterapia paliativa, conforme respectivamente especificado no sub-item "D efinição das M odalidades Terapêuticas".

Estádio IV (M1)

O s dois principais fatores prognósticos para os pacientes em estádio IV são o PS e a perda de peso (maior ou menor que 10\%). 0 sítio de metástase não é fator prognóstico e os pacientes devem ser tratados independentemente da 
metástase ser cerebral, óssea, hepática ou pulmonar.

0 tratamento padrão é a quimioterapia paliativa, associada ou não a radioterapia paliativa do tumor primário ou da lesão metastática.

Em um grupo bem selecionado de pacientes com metástase isolada sincrônica ao diagnóstico do tumor primário, pode ser indicado a cirurgia do tumor primário e a metastatectomia, como no caso de metástase para a glândula supra-renal unilateral ou de metástase cerebral isolada.

Se esta conduta for a escolhida, confirma-se se o paciente não tem acometimento mediastinal ou outrosfocos demetástases, devendo ser incluído na sua avaliação exames de mediastinoscopia e ressonância magnética cerebral.

No caso de metástase supra-renal com indicação cirúrgica, procede-se à quimioterapia neoadjuvante, conforme respectivamente especificado no sub-item "D efinição das M odalidades Terapêuticas".

Situação especial 1 - M etástase única de supra-renal - Realiza-se biópsia ou punção por agulha fina da supra-renal. U ma vez confirmado o diagnóstico de metástase, avalia-se a possibilidade da sua ressecção. Q uando esta for indicada, procede-se à quimioterapia neoadjuvante seguida de ressecção pulmonar.

Situação especial 2 - M etástase cerebral única - Avalia-se a possibilidade de ressecção da metástase cerebral seguida de ressecção pulmonar. Q uando este tratamento é possível, complementa-se com radioterapia paliativa cerebral, conforme respectivamente especificado no sub-item "D efinição das M odalidades Terapêuticas".

\section{SEG UIMENTO}

Após Tratamento com Intenção Curativa

\begin{tabular}{|l|c|c|}
\hline \multicolumn{1}{|c|}{ Procedimento } & $1^{\circ}$ e $2^{\circ}$ ano & $3^{\circ}$ ao $5^{\circ}$ ano \\
\hline História e Exame físico & 3 meses & 6 meses \\
\hline Hemograma completo & 3 meses & 6 meses \\
\hline Fosfatase alcalina e gama GT & 3 meses & 6 meses \\
\hline Radiografia de tórax - em PA e perfil & 3 meses & 6 meses \\
\hline $\begin{array}{l}\text { Tomografia tomputadorizada de } \\
\text { tórax }\end{array}$ & anual & anual \\
\hline
\end{tabular}

Durante e após o Tratamento da Doença

Metastática

D urante o tratamento com quimioterapia paliativa, realiza-se radiografia simples de tórax em PA e perfil, a cada dois ciclos, para avaliação da resposta terapêutica.

Após o tratamento com quimioterapia paliativa (ver respectivamente 0 sub-item "D efinição das M odalidades Terapêuticas"), mantém-se o paciente sob controle clínico e radiológico, com periodicidade sendo definida e mais exames sendo solicitados na dependência da sua sintomatologia.

\section{NOTA FINAL}

Para o IN CA, qualquer conduta aplicada em suas unidades hospitalares que se encontre fora das aqui especificadas, é considerada experimental ou irregular.

\section{BIBLIO G RAFIA}

M inistério da Saúde. Secretaria de Assistência à Saúde. Instituto N acional de Câncer (Brasil). Estimativas da incidência emortalidadepor câncer no Brasil, 2002. Rio deJ aneiro: IN CA; 2002. 90p. $M$ inistério da Saúde. Secretaria de Assistência à Saúde. Instituto N acional deC âncer (Brasil). T N M Classificação dosTumores M alignos. 5a ed. Trad. autorizada pelaU ICC eW iley-Liss deT N M Classification of M alignant Tumours, 1997. IN CA: Rio deJaneiro; 1998. 235p.

Bulzebruck H, Bopp R, D rings P, Bauer E, Krysa $\mathrm{S}$, Probst $\mathrm{G}$, et al. N ew aspects in the staging of lung cancer. Prospective validation of the International Union Against C ancer T N M classification. Cancer 1992;70(5):1102-10.

D illman RO, Seagren SL, Propert KJ, Guerra J, Eaton W L, Perry M C, et al. A randomized trial of induction chemotherapy plus high-doseradiation versusradiation al onein stagelll non-small-cell lung cancer. N Engl J Med 1990;323:940-5.

Feld R, Rubinstein L, Thomas PA. Adjuvant chemotherapy with cyclophosphamide, doxorubicin, and cisplatin in patients with completely resected stage I non-small-cell lung cancer. J N atl C ancer Inst 1993;85:299-306.

Furuse K, Fukuoka M , Kawahara M , N ishikawa $H$, Takada Y, Kudoh S, et al. Phase III study of concurrent versussequential thoracic radiotherapy combination with mitomycin, vindesine, and 
cisplatin in unresectablestagellI non-small-cell lung cancer. J Clin O ncol 1999;17(9):2692-9.

Kearney DJ, Lee TH, Reilly JJ, D eC amp M M, Sugarbaker D J. Assessment of operativerisk in patients undergoing lung resection. I mportance of predicted pulmonary function. Chest 1994;105:753-9.

American Joint Committee on C ancer. Lung. In: AJCC. C ancer staging manual. 5th ed. Philadelphia: Lippincott-Raven; 1997. p. 127-37.

M orton RF, Jett JR, M CG innis WL, Earle JD, Therneau T M , K rook JE, et al. Thoracic radiation therapy alone compared with combined chemoradiotherapy for locally unressectable nonsmall cell lung cancer. A randomized, phasellI trial. Ann Intern M ed 1991;115:681-6.

Pearson FG . N on-small cell lung cancer: role of surgery for stages I-III. Chest 1999; 116(6 Suppl):500S-3S.

Postoperativeradiotherapy in non-small-cell lung cancer: systematic review and meta-analysis of individual patient data from ninerandomised controlled trials. PO RT M eta-analysisTrialists Group. Lancet 1998;352(9124):257-63.

Rapp E, Pater JL, W illan A, Cormier Y, M urray N ,
Evans W K, et al. Chemotherapy can prolong survival in patients with advanced non-small-cell lung cancer: report of a $\mathrm{C}$ anadian multicenter randomized trial. J Clin O ncol 1988;6:633-41.

Rosell R, G omez-Codina J, Camps C, M aestre J, Padille J, C anto A, et al. A randomized trial comparing preoperativechemotherapy plus surgery with surgery alone in patients with non-small-cell lung cancer. N Engl J M ed 1994;330:153-8.

Roth JA, FossellaF, Komaki R, Ryan M B, Putnam $J B J r$, LeeJS, et al. A randomized trial comparing perioperativechemotherapy and surgery with surgery alone in resectable stage IIIA non-small-cell lung cancer. J N atl C ancer Inst 1994;86:673-80. SchaakeK oning $C$, van den Bogaert W, D alesio 0 , Festen J, H oogenhoutJ, van H outteP, et al. Effects of concomitant cisplatin and radiotherapy on inoperablenon-small cell lung cancer. N Engl J M ed 1992;326:524-30.

Wronski M , Arbit E, Burt M , Galicich JH . Survival after surgical treatment of brain metastases from lung cancer: a follow-up study of 231 patients treated between 1976 and 1991. J N eurosurg 1995;83:605-16. 